This item was submitted to Loughborough's Research Repository by the author.

Items in Figshare are protected by copyright, with all rights reserved, unless otherwise indicated.

\title{
Automated registration of SFM-MVS multitemporal datasets using terrestrial and oblique aerial images
}

\section{PLEASE CITE THE PUBLISHED VERSION}

https://doi.org/10.1111/phor.12346

\section{PUBLISHER}

Wiley

VERSION

AM (Accepted Manuscript)

\section{PUBLISHER STATEMENT}

This is the peer reviewed version of the following article: Parente, L, Chandler, J. and Dison, N., (2021). Automated registration of SFM-MVS multitemporal datasets using terrestrial and oblique aerial images. The Photogrammetric Record, 36 (173), pp.12-35, which has been published in final form at https://doi.org/10.1111/phor.12346. This article may be used for non-commercial purposes in accordance with Wiley Terms and Conditions for Use of Self-Archived Versions

\section{LICENCE}

CC BY-NC-ND 4.0

\section{REPOSITORY RECORD}

Parente, Luigi, Jim Chandler, and Neil Dixon. 2021. "Automated Registration of SFM-MVS Multitemporal Datasets Using Terrestrial and Oblique Aerial Images". Loughborough University. https://hdl.handle.net/2134/13353245.v1. 


\title{
AUTOMATED REGISTRATION OF SFM-MVS MULTITEMPORAL DATASETS USING TERRESTRIAL AND OBLIQUE AERIAL IMAGES
}

\author{
LUIGI PARENTE* (L.Parente@unimore.it) \\ Department of Engineering (DIEF) 'Enzo Ferrari', University of Modena and Reggio \\ Emilia, Modena, Italy \\ JIM H. CHANDLER (JimChandler.Spatial@virginmedia.com) \\ NEIL DiXON (N.Dixon@lboro.ac.uk) \\ School of Architecture, Building and Civil Engineering, Loughborough University, UK \\ * Corresponding author
}

\begin{abstract}
Accurate alignment of $3 D$ models is critical for valid change-detection analysis from multitemporal photogrammetric datasets. This paper assesses an automated registration strategy which uses the scale-invariant feature transform (SIFT) algorithm implemented in modern photogrammetric software. This registration solution, also known as 'Time-SIFT', was tested at two study sites featuring vertical surfaces, including a sea cliff $\left(\sim 500 \mathrm{~m}^{2}\right)$ and a quarry face $\left(\sim 50000 \mathrm{~m}^{2}\right)$. Tests demonstrated that the investigated registration strategy can achieve accurate alignments between multitemporal point clouds even when using multisource and multi-perspective data, captured across widely varying spatial and temporal scales and under a range of weather and illumination conditions. The combination of the Time-SIFT approach with an ICP algorithm produced moderate improvements in the alignment. Furthermore, the use of an innovative direct georeferencing technique, that used the tracking feature of a robotic total station, allowed for accurate georectification of $3 D$ models.
\end{abstract}

KEYWORDS: photogrammetry, structure from motion (SfM), image-based point cloud, registration, Time-SIFT, PhotoModeler software

\section{INTRODUCTION}

THE USE OF STRUCTURE-FROM-MOTION (SfM) PHOTOGRAMMETRY (Snavely et al., 2006) coupled with multiview stereo (MVS) algorithms (Seitz et al., 2006) has undoubtedly become a commonly used non-invasive surveying technique for a wide range of geoscientific applications including coastal morphology, soil science, glaciology, fluvial morphology and many others (Eltner et al., 2016). Many authors have given a detailed description of the SfM-MVS technique (for example James and Robson, 2012; Smith et al., 2015). The main advantages of this technique are related to the use of a varied range of cheap sensors (such as digital single-lens reflex (DSLR), action and trail cameras) and platforms (tripods, poles, unmanned aerial vehicles (UAVs)), user-friendly software (both commercial and open-source) and mostly automated processing steps. Furthermore, this photogrammetric technique is flexible and can be applied to a wide range of scales. These advantages and results comparable to those achieved by higher priced surveying methods (for example terrestrial laser scanners: Thoeni et al., 2014; Chandler and Buckley, 2016) has fascinated both geoscientists and non-expert users. Although most of the SfM-MVS photogrammetry workflow is standardised and can return high-quality 3D models, some steps (such as image acquisition and camera calibration) still require a skilled operator to 
identify potential sources of error and take appropriate decisions in order to avoid the generation of inaccurate metric information (Eltner et al., 2016; Mosbrucker et al., 2017; James et al., 2019).

When conducting change analysis using multitemporal 3D datasets, the establishment of a common and stable reference system is crucial. In fact, the correct co-registration of photogrammetric outputs (such as point clouds) is a delicate step to carry out in order to minimise the distance between corresponding points and reduce the introduction of errors (Micheletti et al., 2014; Scaioni et al., 2015). Depending on the application, a range of strategies are generally adopted to minimise errors in the similarity transformation (rotation, translation and scaling) applied to the multitemporal 3D models (for example Zang et al., 2019; Cucchiaro et al., 2020). A detailed description of registration approaches is provided by Al-Rawabdeh et al. (2016).

The standard and most widely used registration strategy for photogrammetric projects includes placing appropriately distributed targets or identifying natural and/or artificial features in the study area (for example Westoby et al., 2012; Esposito et al., 2017). In either case, these ground control points (GCPs) must be stable over the monitoring period or their coordinates re-established in the same coordinate system. Their positions can be measured using accurate instrumentation (such as a real-time kinematic differential Global Navigation Satellite System (RTK-dGNSS) or total station) and used to georeference the 3D model (Brunier et al., 2016; Esposito et al., 2017). A frequently used approach to reduce alignment errors due to manual misidentification of common GCPs is the iterative closest point (ICP) algorithm (for example Lague et al., 2013; Micheletti et al., 2014; Cucchiaro et al., 2020). The efficiency of the ICP algorithm is reduced when applied to point clouds with large voids or where real substantial change has occurred. An option used to maximise the quality of the ICP registration is applying the algorithm only to stable zones, thus isolating areas where change may have occurred (Turner et al., 2015).

When the use of GCPs is not feasible, an option could involve the use of a fixedcamera set-up, which has the theoretical advantage of generating models aligned in the same local reference system (Travelletti et al., 2010). Another option could be direct georeferencing, which involves the measurement of camera position/orientation to georeference the generated 3D models (Turner et al., 2014; Armstrong et al., 2018). AlRawabdeh et al. (2016) proposed a robust registration option for UAV imagery that relies on the knowledge of the coarse image position at each exposure. However, for terrestrial applications, measuring ground-based camera positions/orientations repeatedly over time would be time-consuming and hence costly. Furthermore, determining camera position/orientation is challenging as the perspective centre of a lens is not accessible. Thus, the challenges described (maintaining cameras in fixed position/orientation; measuring the camera position/orientation for each epoch; installing and surveying an appropriate number of targets at each epoch; and then requiring the ability (and time) of the operator to identify the same stable features in multitemporal point clouds) have led to a desire to identify alternative registration solutions.

In response to this, the research presented in this paper has investigated a quick and low-cost solution that involves the use of the scale-invariant feature transform (SIFT) algorithm (Lowe, 2004) across multiple photographic epochs. To the authors' knowledge, this approach was first adopted to compute the $2 \mathrm{D}$ registration between historical and modern aerial images (Fox and Cziferszky; 2008; Jao et al., 2014). Similarly, Chen and Tseng (2016), georeferenced historical aerial photography and satellite imagery by adopting the SIFT algorithm to extract common image features and the random sample consensus (RANSAC) algorithm to remove outliers (wrong point matches).

A similar approach that exploits the automated SfM-MVS pipeline available in modern photogrammetric software can be adopted to automatically generate aligned multitemporal 2D and 3D datasets (for example Vargo et al., 2017; Feurer and Vinatier, 2018). This registration approach involves the use of multi-epoch datasets in one single photogrammetric block. Optionally, just one georeferenced dataset is necessary to determine the exterior camera parameters (including camera location) of all epochs (Vargo et al., 2017) and to generate aligned dense point clouds or digital elevation models (DEMs) (Feurer and Vinatier, 2018). This innovative 3D registration method was first identified as 'Time-SIFT' by Feurer and Vinatier (2018) who demonstrated its potential for producing DEMs of difference (DoD) from multitemporal aerial archive images, suitable for landscape change analysis. Other analogous recent studies have highlighted the potential 
of such an approach to co-register multitemporal images captured from UAV (AlRawabdeh et al., 2016; Cook and Dietze, 2019) and terrestrial camera stations (Filhol et al., 2019). However, this registration approach was not adopted to investigate the ability to register multitemporal epochs captured from both terrestrial and UAV-based cameras into a common reference system. Integration of multisource/perspective images (for example merging aerial and terrestrial set of images captured with oblique and nadir views) can offer a promising solution to achieve full photogrammetric reconstruction of complex environments (Nex et al., 2015). Furthermore, the benefits of combining the SIFT-based approach with an ICP algorithm were not examined. Although a few case studies have already investigated the ability of the Time-SIFT registration strategy (Feurer and Vinatier, 2018; Cook and Dietze, 2019; Filhol et al., 2019; Feurer et al., 2020), its robustness for a wider range of imaging conditions, in particular the use of aerial oblique and terrestrial imagery, must be further investigated.

The purpose of this paper is primarily to investigate the capabilities of the Time-SIFT method to co-register SfM-MVS-based point clouds using photographic datasets captured from different sensors and platforms (thus allowing for image acquisition with different viewing geometry). The investigated registration solution was tested using datasets collected at two study sites with different scales and characteristics. The sets of images were captured at widely varying temporal intervals under a range of weather and illumination conditions. Furthermore, the use of the ICP co-registration algorithm was assessed with models already aligned using Time-SIFT. In addition, an innovative direct georeferencing technique that combines UAV photogrammetry and a total station survey was also implemented, using the Time-SIFT solution, to provide an alternative to traditional registration approaches in the absence of GCPs.

\section{STUDY SITES}

The photographic datasets were collected at two sites which differ in a range of aspects, including geological and structural features, instability triggers, accessibility and size. However, both study sites are characterised by a near-vertical slope. The first site is a sea-cliff section ( $25 \mathrm{~m}$ x $20 \mathrm{~m}$ ) known as the Spittles Cliffs, situated east of Lyme Regis $\left(50.72866^{\circ} \mathrm{N}, 2.92840^{\circ} \mathrm{W}\right)$, England (Fig. 1(a)) and easily accessible from the adjacent sea defences and promenade. More details about this cliff section can be found in Parente et al. (2019).

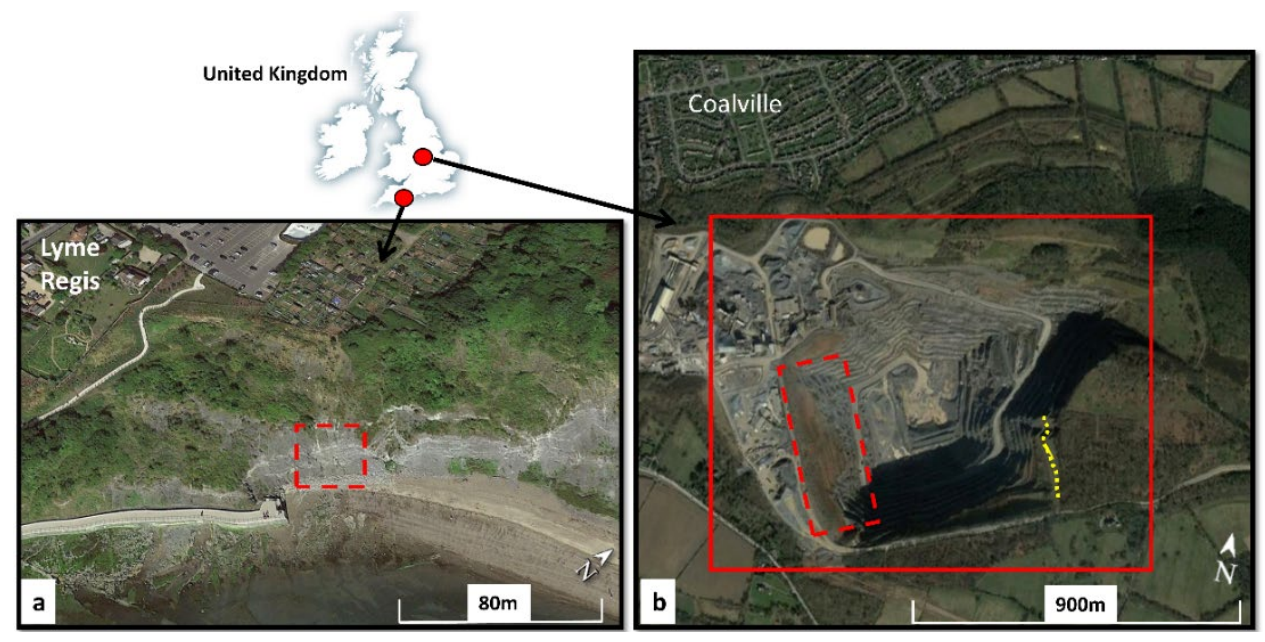

FIG. 1. Overview of the two study sites. (a) Lyme Regis sea-cliff: the red dashed rectangle shows the position of the investigated coastal cliff. (b) The two red rectangles show the Bardon Hill Quarry (solid line) and the monitored outcrop (dashed line). The dashed yellow line indicates the path from where ground-based photographic datasets were acquired.

The second study site permitted investigation over a larger area and from a greater distance. This site is a large and active quarry outcrop known as 'Bardon Hill Quarry', located southeast of Coalville $\left(52.71202^{\circ} \mathrm{N}, 1.32763^{\circ} \mathrm{W}\right)$, England (Fig. 1(b)). The area has a long tradition of quarrying due to the valuable properties of the local rock (Burton, 
1622), which is mostly used in construction. The geology of Bardon Hill Quarry is characterised by a late Precambrian (Ediacaran period) assemblage of igneous and metasedimentary rocks (known as 'Bardon Breccia'), which form the 'core' of the quarry (Carney, 2010). The quarry reaches a maximum lateral extension of approximately $1000 \mathrm{~m}$ (measured across the top of the quarry as an Euclidean distance). The quarry floor is at approximately $-30 \mathrm{~m}$ AOD (above Ordnance Datum) and is thus below sea level. The excavation reaches a maximum depth of approximately $305 \mathrm{~m}$ (July 2019). The selected monitored area is approximately $250 \mathrm{~m}$ x $200 \mathrm{~m}$ and has a history of minor failures. The upper part is characterised by a thin layer of glacial till and alluvium. However, the slope geology is predominated by the presence of mudstones and the Bardon Breccia, which cause slope instability. Both lithologies are divided into quarried cliff faces separated by small benches. The slope angle of the mudstones faces vary between approximately $42^{\circ}$ and $60^{\circ}$ (the overall slope angle is about $39^{\circ}$ ). The Bardon Breccia faces have an individual slope angle of $70^{\circ}$ (with an overall slope angle of about $45^{\circ}$ ).

\section{METHODS}

\section{Dataset Acquisition - First Site (Cliff)}

The fieldwork on the first study site (indicated as 'Cliff') provided the opportunity to acquire photographic datasets on two successive days in August 2017. A summary of information related to the data collection carried out at the Cliff site are shown in Table I. More details about the approach used to acquire each dataset is provided in Parente et al. (2019). In summary, on 22nd August 2017, eight 'ringed automatically detected' (RAD) targets were temporarily positioned across the site of interest (Fig. 5). The RAD targets were used to facilitate automatic processing operations in PhotoModeler (PhotoModeler, 2019). Their position was measured using a reflectorless total station to establish control in a local reference system. Then, a low-priced DSLR camera (Nikon D80 - 10 Mpixel sensor with 3872 x 2592 pixels) mounted on a tripod, was used to acquire four images of the site of interest. A Nikkor lens with fixed focal length of $24 \mathrm{~mm}$ was used. A constant camerato-object distance $(\sim 30 \mathrm{~m})$ and mildly convergent configuration was ensured. With this configuration, the four images of each epoch captured the entire portion of cliff under investigation (yellow area in Fig. 5). The corresponding ground sampling distance (GSD) for the DSLR-based acquisition was about $7.6 \mathrm{~mm}$.

On the same day, a UAV-based photogrammetric survey (DJI Phantom Professional 3 with integrated camera: $20 \mathrm{~mm}$ (35 mm equivalent) $\mathrm{f} / 2.8$ lens coupled with a $6.2 \times 4.6 \mathrm{~mm}$ sensor) was also conducted, consisting of a dataset of 29 images (indicated as 'Cliff1UAV'). The flight route was controlled manually and the photographs were acquired from a range of positions flying approximately $30-35 \mathrm{~m}$ from the cliff at a flight altitude of between 10 and $25 \mathrm{~m}$ (average GSD of $14 \mathrm{~mm}$ ). Acquisition was conducted by combining oblique (maximum deviation from the horizontal plane of $30^{\circ}$ ) and perpendicular (to the cliff face) images, whilst maintaining a mildly convergent configuration.

The following day, only a photographic ground-based acquisition approach was adopted whilst retaining the same camera geometry used the day before. The two groundbased datasets, collected on 22nd and 23rd August 2017, are indicated as 'Cliff1' and 'Cliff2', respectively.

TABLE I. Summary of information relative to the datasets collected at the first site (Lyme Regis, UK).

\begin{tabular}{ccccc}
\hline Date & Datasets & Acquisition sensor & Targets & Dataset IDs \\
\hline \multirow{2}{*}{$22 / 08 / 17$} & 4 ground-based photos & Nikon D80 $(24 \mathrm{~mm})$ & Yes & Cliff1 \\
\cline { 2 - 5 } & 29 UAV-based photos & DJI Phantom 3 Professional & Yes & Cliff1-UAV \\
\hline $23 / 08 / 17$ & 4 ground-based photos & Nikon D80 (24 mm) & No & Cliff2 \\
\hline
\end{tabular}




\section{Dataset Acquisition - Second Site (Quarry)}

Data collection at the Bardon Hill Quarry test site (indicated as 'Quarry') started in November 2018. Ground-based photogrammetric surveys were conducted over a time span of nine months and included a total of ten photogrammetric epochs (indicated as 'Quarry1', 'Quarry2', ..., 'Quarry 10'). A summary of the surveys (and dates) conducted at this site is reported in Fig. 2. All photogrammetric datasets were collected at various times during the day (between 9am and 2pm) during the different seasons and, inevitably, experienced a range of weather, atmospheric and illumination conditions (Table II).

Each epoch comprised of four images captured with the Nikon D80 DSLR, mounting a $35 \mathrm{~mm}$ lens, with a tripod and a remote shutter release used to acquire imagery free of blur. Each image was captured with the camera axis perpendicular to the site and from an acquisition zone that was opposite the photographed site (Figs. 1(b) and 3(b)). The approximate distance between the quarry face and the camera locations was $700 \mathrm{~m}$, achieving a GSD of about $120 \mathrm{~mm}$. Initially, photogrammetric datasets (from Quarry1 to Quarry5) were captured by moving the tripod to four positions identified by markers (Fig. 3(a)). Quarry6 to Quarry8 were collected from stationary and assumed 'fixed' camera stations, provided by fixing standard tripods to metal poles that were drilled about $70 \mathrm{~cm}$ into the ground (Fig. 3(b)). However, a visual inspection of the datasets collected for epoch Quarry8, highlighted imperfect image overlap, suggesting a slight change in orientation (probably due to wind, animal/bird interference or ground deformation) for two of the four camera stations. Images from the last two epochs (Quarry9 and Quarry10), were captured by trying to locate the camera as close as possible to the original 'fixed' positions, because the camera mounts had been vandalised and were unusable (Fig. 3(c)). This overly simplistic camera mounting strategy led to small changes in orientation for all camera stations. Thus, long-term acquisition from truly fixed camera stations proved difficult to achieve, requiring a Time-SIFT registration approach to be investigated.

TABLE II. A summary of the illumination conditions at the time of acquisition of each epoch. (*based on the acquisition of the first photo).

\begin{tabular}{cccccc}
\hline Dataset IDs & Date & Time* & Weather condition & $\begin{array}{c}\text { Illumination } \\
\text { quality }\end{array}$ & $\begin{array}{c}\text { Shutter } \\
\text { speed }\end{array}$ \\
\hline Quarry1 & $5 / 11 / 18$ & $13: 17$ & Cloudy and foggy & Poor & $1 / 50 \mathrm{~s}$ \\
\hline Quarry 2 & $15 / 11 / 18$ & $12: 26$ & Mostly cloudy and foggy & Poor & $1 / 50 \mathrm{~s}$ \\
\hline Quarry 3 & $7 / 12 / 18$ & $12: 05$ & Cloudy and light rain & Poor & $1 / 30 \mathrm{~s}$ \\
\hline Quarry 4 & $11 / 01 / 19$ & $10: 49$ & Cloudy and foggy & Very Poor & $1 / 20 \mathrm{~s}$ \\
\hline Quarry 5 & $15 / 02 / 19$ & $13: 46$ & Mostly cloudy and foggy & Very Poor & $1 / 60 \mathrm{~s}$ \\
\hline Quarry 6 & $19 / 03 / 19$ & $12: 33$ & Sunny & Good & $1 / 40 \mathrm{~s}$ \\
\hline Quarry 7 & $10 / 04 / 19$ & $11: 44$ & Mostly sunny & Very good & $1 / 250 \mathrm{~s}$ \\
\hline Quarry 8 & $2 / 05 / 19$ & $11: 29$ & Mostly sunny & Very good & $1 / 125 \mathrm{~s}$ \\
\hline Quarry 9 & $17 / 05 / 19$ & $09: 05$ & Cloudy & Good & $1 / 40 \mathrm{~s}$ \\
\hline Quarry 10 & $19 / 07 / 19$ & $10: 04$ & Cloudy & Good & $1 / 40 \mathrm{~s}$ \\
\hline
\end{tabular}

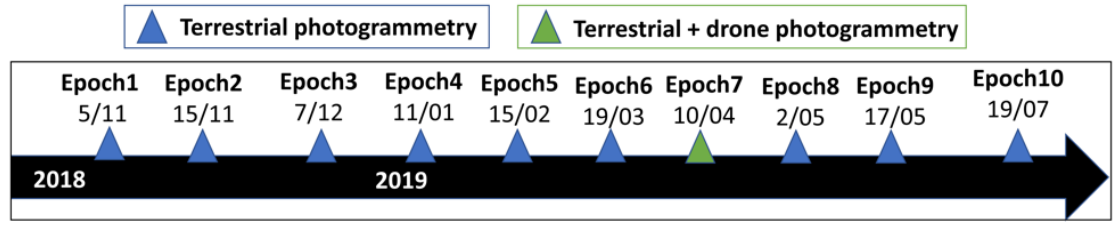

FIG. 2. Timeline of datasets acquisition dates for the quarry site from November 2018 to July 2019.

\section{Tracking Solution for Direct Georeferencing at Quarry Site}

Due to the difficulties in installing and surveying a suitable number of targets within the Quarry site, the necessary real-world and absolute coordinates were provided by an innovative 'direct' solution. On 10th April 2019 an additional photogrammetric dataset of the Quarry was collected in clear atmospheric conditions, using a DJI Phantom 3 Professional quadcopter. The UAV-based dataset is indicated as 'Quarry7-UAV'. Single oblique images (with the camera axis up to $30^{\circ}$ from the horizontal but orthogonal to the 
quarry face) were captured from eight locations with the UAV hovering at an altitude between 190 and $215 \mathrm{~m}$ and with a constant camera-to-object distance of about $400 \mathrm{~m}$ (GSD of $173 \mathrm{~mm}$ ). The eight images captured the full extent of the study site.

Before flying the UAV, a retroreflector prism (Leica mini 360, GRZ101) was installed in the lower part of the UAV. This multi-directional $360^{\circ}$ prism, combined with a robotic total station, permitted real-time measurement of the position of the UAV. This was achieved by exploiting the "tracking" mode function of the total station that allows position measurement of a moving retroreflector prism. The small offset between the prism and the perspective centre of the camera lens was not accounted for as the $70 \mathrm{~mm}$ offset was small at this photo-scale. To relate the UAV imagery to the national mapping framework in the UK (Ordnance Survey OSTN15), the position of the total station was determined using Trimble virtual reference station (VRS) technology (a commercial network and real time kinematic solution that uses a single survey-grade GNSS receiver); a second tripod and prism was setup approximately $500 \mathrm{~m}$ away, with its OSTN15 grid coordinates determined using the VRS system. Additionally, two prisms (IDs: 103 and 104) were set up on tripods positioned on the bund wall of the haul road at the bottom of the investigated site and used as check points. Then, the position of these points (centre of the prism) were computed by taking conventional angle circle readings and distance measurements using the total station (accuracy of $1 \mathrm{~mm} \pm 2 \mathrm{ppm}$ ), all in the OSTN15 grid coordinate system. The 'true' position (computed through the survey) of the check points were used to estimate the accuracy of the positional estimate derived from the photogrammetry-based measurement.

During the UAV-based image acquisition, two operators were needed to efficiently synchronise the photo shooting and the recording of the position of the UAV (centre of the Leica mini 360). This was achieved using simple audio communication (such as counting to three). This approach ensured that the two operators triggered the two remote controls (for both the total station and hovering UAV camera) with a temporal offset below one second.

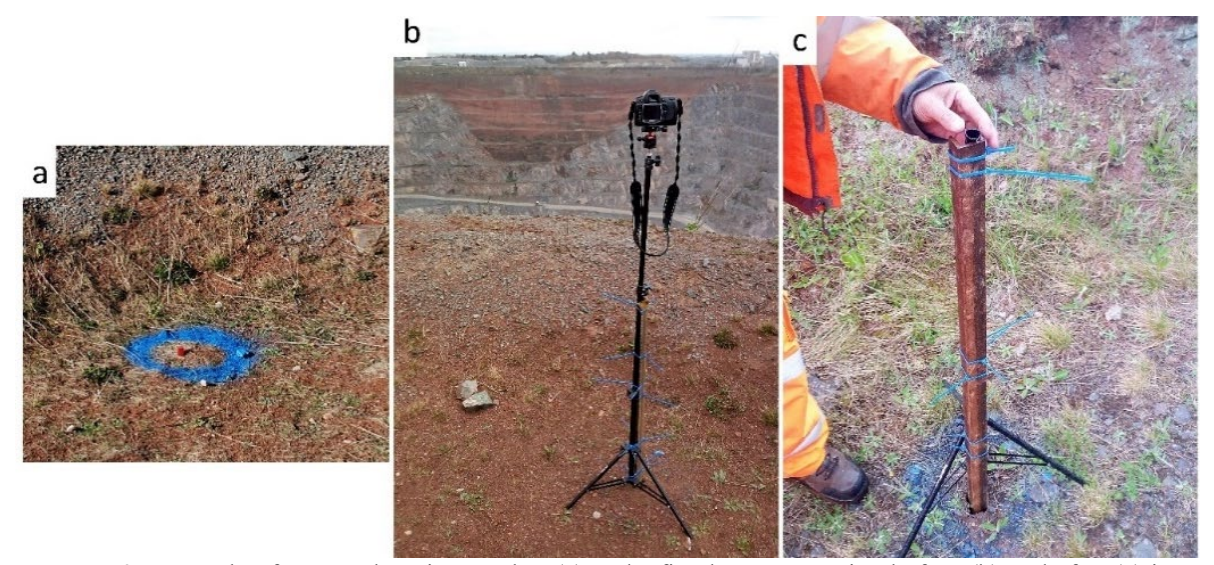

FIG. 3. Example of camera location marker (a) and a fixed camera station before (b) and after (c) it was vandalised.

\section{Camera Calibration}

When images are acquired from variable locations, the adoption of a convergent camera configuration coupled with accurately estimated interior orientation parameters can eliminate challenges posed by systematic errors in SfM-related applications (Parente et al., 2019). For both test sites (Cliff and Quarry), following acquisition of the first epoch, the cameras used to collect the ground-based and aerial imagery were calibrated by capturing 12 images of a calibration wall with 210 coded targets (Fig. 4). The size of the calibration wall allowed images to be acquired from an adequate distance and the same camera and lens settings used in the field were maintained during the calibration phase. Calibration images were captured from convergent positions, with large horizontal and vertical bases, and included shots obtained by rotating the camera around its optical axis $\left(90^{\circ}\right.$ anticlockwise), as suggested by Gruen and Beyer (2001). Images were then uploaded to the Camera Calibrator (a PhotoModeler tool), which provides a largely automated solution to 
detect, identify and measure RAD targets and to estimate sensor calibration parameters. This procedure achieved a low overall residual RMS $(<0.1$ pixel) and low maximum point mark residual $(<1$ pixel), which suggested suitability of the estimated calibration parameters to extract spatial data of an appropriate quality.

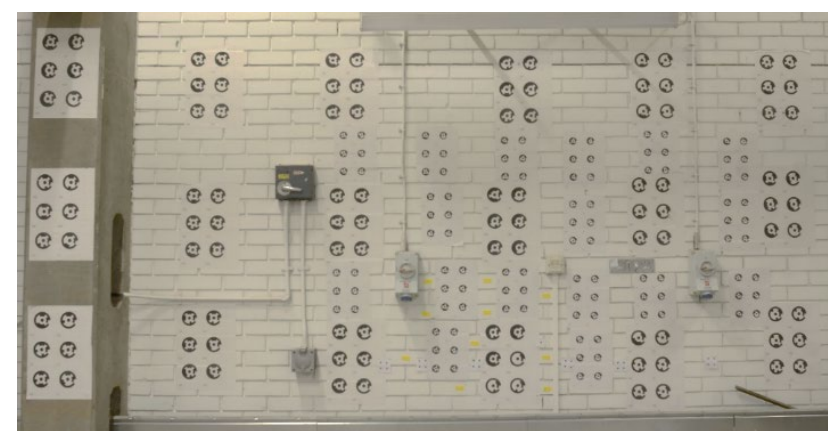

FIG. 4. RAD targets attached to a laboratory wall to provide a large calibration field identified as 'calibration wall'.

\section{Dense Model Generation and Registration}

The photogrammetric datasets of both sites (Cliff and Quarry) were processed in PhotoModeler Premium version 2019.1.2.2469 (PhotoModeler, 2019) to generate SfMMVS-based dense models. PhotoModeler is commercial software that represents a complete solution for photogrammetric reconstruction with features similar to other professional software that have been more commonly adopted by the geoscientific community (such as Agisoft PhotoScan/Metashape, Autodesk 123D Catch, VisualSFM, and so on: Thoeni et al., 2014; Micheletti et al., 2014; Mosbrucker et al., 2017).

Two slightly different SfM-MVS workflows were adopted in this paper, indicated as 'single epoch' and 'multi-epoch'. The single epoch workflow is the classical approach found in SfM-based literature. This involves working with a single block of images at a time with each epoch processed separately, so creating multiple photogrammetric projects (see, for example, Prosdocimi et al., 2015). With this approach, all photographs from a single epoch are imported into the software and the previously estimated and stored camera calibration file is selected. The next automated step is the estimation of camera pose and photos orientation via the 'SmartMatch' tool in PhotoModeler. This step determines matching features across the images using a SIFT algorithm, and subsequently an iterative bundle adjustment generates an initial 3D model with relatively few object points (a sparse point cloud). Then, the model density is increased via the MVS algorithms. If GCPs are visible and their position is measured (for example using a total station), their coordinates are used to georeference the photogrammetric model.

The single epoch workflow was adopted solely for the photogrammetric datasets collected at the Cliff site and not at the Quarry. Specifically, the Cliff1 epoch was used to produce a 'reference' model that was scaled, rotated and translated to the same geometry of GCPs (RAD targets) estimated with the total station survey. Two additional dense models were generated adopting the single epoch workflow with the Cliff2 and Cliff1-UAV datasets (indicated as 'compared models'). The two compared models were initially generated in an arbitrary coordinate system and without scaling. In order to align the three dense models (Cliff1, Cliff2 and Cliff1-UAV) they were imported into CloudCompare version 2.10.2 (CloudCompare, 2019), a popular open-source software package commonly used by SfM-MVS and TLS users (see Lague et al., 2013; Micheletti et al., 2014; Prosdocimi et al., 2015). The latter software was used to estimate a 3D similarity transformation between the reference and the compared models. This involved the identification and manual selection of well-distributed homologous points (tie points) between the refence and the compared models. A total of five stable tie-point pairs were selected (their position is shown in Fig. 5). In addition to the point-pair solution (indicated as 'manual' registration), the iterative closest point (ICP) algorithm implemented in CloudCompare (Besl and McKay, 1992) was used to optimise the initial registration. This registration approach is indicated as 'manual+ICP'. 


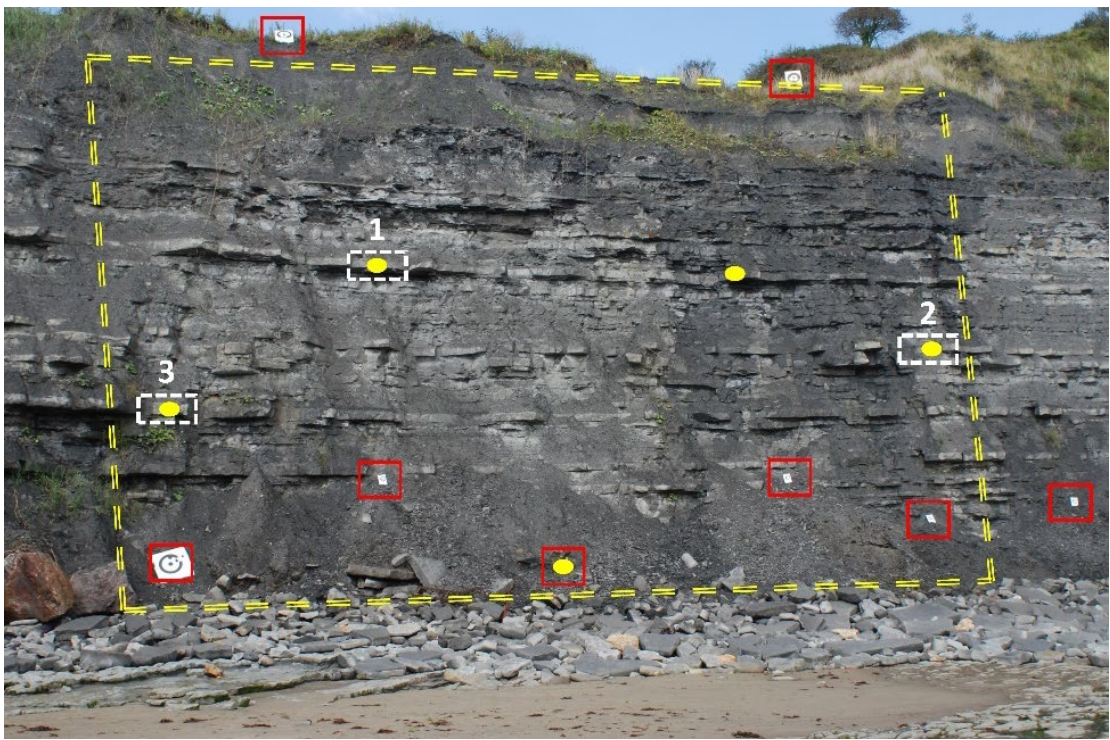

FIG. 5. First site at Spittles Cliffs, Lyme Regis, UK. Spatial distribution of eight RAD targets (red squares), three small stable areas (white dashed rectangles) and five tie-points used for manual registration (yellow dots). The yellow dashed rectangle indicates the areal extension of the cliff considered for analysis.

The multi-epoch workflow, corresponding to the investigated Time-SIFT solution, involves joining all multitemporal photographic epochs in a single photogrammetric project (Feurer and Vinatier, 2018) and was used with datasets collected at both sites, Cliff and Quarry. The multi-epoch approach involves the following series of steps that can be fully automated in a programming environment (MATLAB, Python, and so on), although this is not essential:

Step 1: Generating the sparse point cloud of the reference model is the first step. For this purpose, the block of georeferenced images was used (the Cliff1 and Quarry7-UAV were used as georeferenced datasets for the Cliff and Quarry photogrammetric projects, respectively).

Step 2: Successively, the remaining multitemporal images were imported into the photogrammetric project and 'SmartMatch' was run to automatically detect and match stable features across all photographs from all epochs to orientate the entire set of photographs through time. This step is critical as 'SmartMatch' automatically removes potential matching points that have changed (for example due to displacements on the monitored slope) and so preventing misorientation due to mismatched points.

Step 3: The 'Process' tool in PhotoModeler, which performs a bundle adjustment, is used to establish the sparse point clouds and camera station positions and orientations in the same coordinate system.

Step 4: Lastly, the multitemporal images are separated into single photo collections representing each photographic epoch. It is then possible to apply just the MVS algorithm to generate dense point clouds representing each individual epoch.

Using this procedure, all dense point clouds through time are automatically generated in the same common reference system. This approach therefore neatly registers all epochs to the coordinate system of the reference model. The addition of the ICP algorithm to the point clouds which have already been aligned using Time-SIFT provided a further registration strategy indicated as 'Time-SIFT+ICP'.

The settings used for the ICP and MVS algorithms remained constant for both tests (Cliff and Quarry). Specifically, for the ICP algorithm, a final overlap of 95\% was selected along with the 'adjust scale' option. The default settings were maintained for the other ICP parameters. The parameters used for the MVS algorithms are the same indicated in Parente et al. (2019).

In summary, a total of four different registration strategies were used, namely: manual; manual+ICP; Time-SIFT; and Time-SIFT + ICP. The manual and manual + ICP registrations obtained with the single epoch workflow were adopted solely for the Cliff work. The TimeSIFT and Time-SIFT+ICP strategies, obtained with the multi-epoch workflow, were 
adopted for both the Cliff and the Quarry datasets. The single epoch approach was not adopted to register the Quarry datasets because of the difficulties in installing RAD targets (logistical constraints and safety issues) and clearly identifying stable 3D common features over multitemporal datasets with a coarse resolution.

\section{Comparison of Registration Strategies}

The datasets collected at the Cliff site offered the opportunity to compare alignments obtained with both registration workflows, including the single epoch and multi-epoch approaches. The Cliff1 dense point cloud was determined in the local coordinate system defined by the visible GCPs. The Cliff1-UAV and Cliff2 models were then aligned to the local system using both the single epoch and multi-epoch approaches.

An evaluation of the different registration approaches was carried out by computing the $\mathrm{M} 3 \mathrm{C} 2$ (multiscale model-to-model cloud comparison - a plugin available in CloudCompare and described by Lague et al. (2013)). Deviations were generated by comparing the full extent of 3D reconstructions captured on the same day (Cliff1 and Cliff1-UAV) and the point clouds of three small stable areas obtained on different dates (Cliff1 and Cliff2). The extension and location of the full 3D reconstruction and small stable areas are shown in Fig. 5. The M3C2 comparison between the full 3D reconstructions of Cliff1 and Cliff1-UAV was repeated four times:

(1) the point clouds were aligned through a manual approach only;

(2) the manual+ICP registration was implemented;

(3) a Time-SIFT strategy was employed; and

(4) a Time-SIFT+ICP registration.

The same four registration approaches were used for the M3C2-based comparisons of the three stable areas of Cliff1 and Cliff2.

A similar registration assessment method was adopted for the datasets collected at the Quarry site. However, for this study site only the multi-epoch approach (Time-SIFT and Time-SIFT+ICP) was evaluated due to the absence of targets and clearly identifiable stable features that prevented the use of a manual registration. The assessment was carried out by computing signed differences ( $\mathrm{M} 3 \mathrm{C} 2$ tool) generated by comparing:

(1) two datasets captured on the same day (full extent of Quarry7 and Quarry7-UAV - Fig. 6); and

(2) ten multitemporal datasets generated over nine months. The multitemporal analysis was conducted on a small stable area (Fig. 6) that was cropped from each ground-based point cloud (from Epoch1 to Epoch10) and compared with the corresponding area cropped from the UAV-based reconstruction.

For each pair of co-registered point clouds, the quality of the alignment solution was determined by estimating the mean distance $(\mu)$ and standard deviation $(\sigma)$ within stable areas.

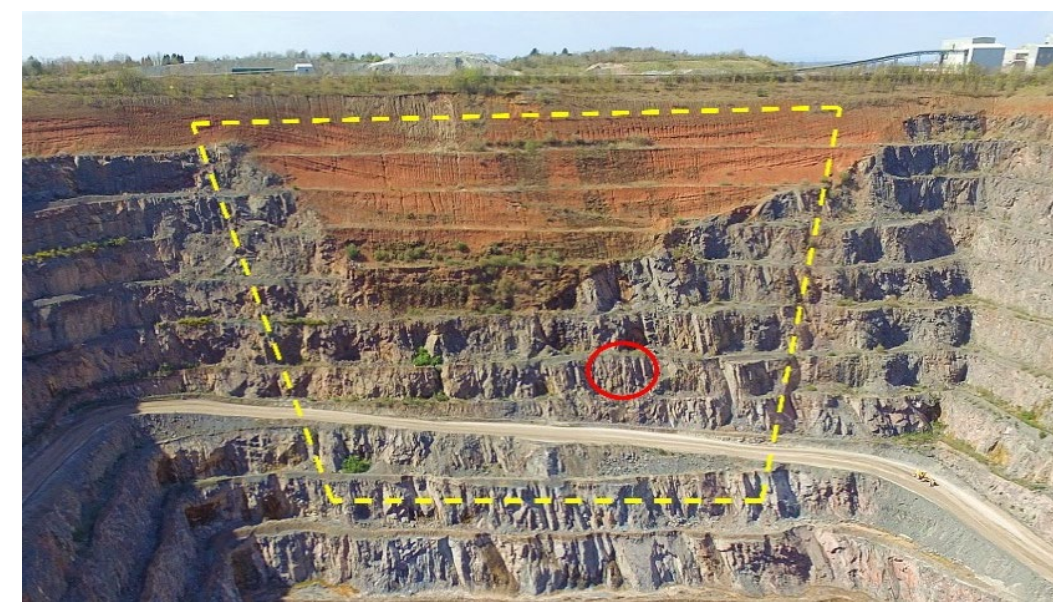

FIG. 6. Second site at Bardon Hill Quarry, Coalville, UK. The dashed yellow rectangle indicated the full extent of the Quarry site of interest. The red circle indicates the location of the area considered inactive during the observation period (November 2018 - July 2019). 


\section{RESUltS}

Site 1: Sea Cliff (Lyme Regis, UK)

Fig. 7 conveys graphically the $\mathrm{M} 3 \mathrm{C} 2$ distances for the four co-registration strategies (manual, manual+ICP, Time-SIFT and Time-SIFT $+\mathrm{ICP}$ ) across the full extent of the Cliff site (models compared are Cliff1 and Cliff1-UAV). As expected, the manual registration produced the largest differences $(0.016 \mathrm{~m}$ and $0.082 \mathrm{~m}$ in terms of mean distance $(\mu)$ and standard deviation $(\sigma)$, respectively) but this decreased significantly after adopting the ICP algorithm $(\mu=-0.001 \mathrm{~m}$ and $\sigma=0.040 \mathrm{~m})$. In contrast, the multi-epoch alignment produced similar distance statistics with or without the adoption of the ICP algorithm, with mean distances varying between 0.001 and $0.006 \mathrm{~m}$ and standard deviations between 0.038 and $0.040 \mathrm{~m}$, respectively. However, a modest improvement is discernible when the ICP algorithm was included (about $5 \mathrm{~mm}$ in terms of mean distance and about $3 \mathrm{~mm}$ in standard deviation). Observing the spatial distribution of change illustrated in Fig. 7 it is evident that the manual registration (Fig. 7(a)) produces a distinct pattern of discrepancies when compared to the other representations (Fig. 7(b), (c) and (d)). In fact, the Time-SIFT and ICP-based approaches generated positive and negative differences that are more homogeneously distributed when compared to the manual registration. Furthermore, adopting a manual procedure (Fig. 7(a)) produced sharp transitions between positive (reddish colours) and negative (blueish colours) change points. A more gradual transition (pale hues and white colours) is observed with the other representations.

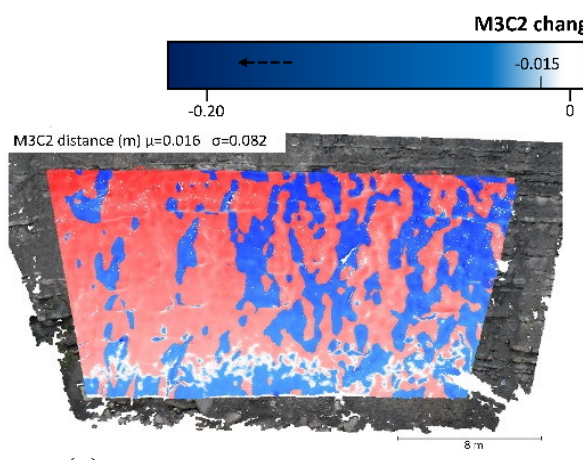

(a)

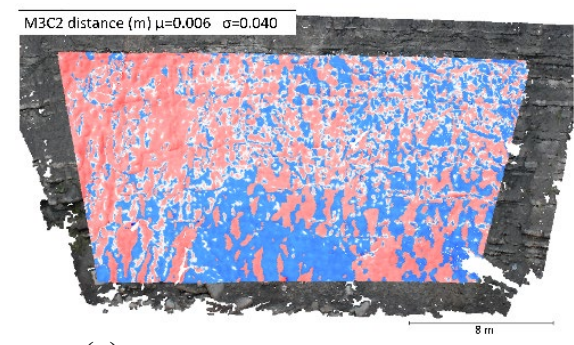

(c)

FIG. 7 Spatial distribution of M3C2 error distances estimated between the UAV (Cliff1-UAV) and terrestrial (Cliff1) point clouds acquired the same day when co-registered with (a) manual, (b) manual+ICP, (c) TimeSIFT and (d) Time-SIFT+ICP strategies.

The manual, manual+ICP, Time-SIFT and Time-SIFT+ICP registration approaches were also evaluated by comparing the point clouds obtained on different dates with a ground-based approach (Cliff1 and Cliff2). In this case analyses were carried out solely within three small areas considered stable (Fig. 5), to avoid inclusion of real morphological change in the statistics. For consistency with the previous analysis (Fig. 7), the differences between point clouds were estimated using the M3C2 tool. Statistical results of the M3C2 comparisons (Table III) show again that the largest differences are estimated for the manually registered clouds. More comparable results are derived using the other three registration approaches. The Time-SIFT + ICP and the manual + ICP approaches produced values with differences under $5 \mathrm{~mm}$ in terms of standard deviation of the three stable areas. 
Applying the ICP to the clouds already registered by Time-SIFT appeared beneficial in terms of a decrease in standard deviations values, further reducing the distances between point clouds. Summarising Table III, tests on the three stable areas reveal standard deviation values in the following ranges:

- $\quad 0.028-0.046 \mathrm{~m}$ for the manual registration;

- $\quad 0.008-0.009 \mathrm{~m}$ for the manual+ICP registration;

- $\quad 0.009-0.017 \mathrm{~m}$ for the Time-SIFT registration; and

- $\quad 0.007-0.013 \mathrm{~m}$ for the Time-SIFT+ICP registration.

These values clearly demonstrate only very minor differences between the manual+ICP and both Time-SIFT-based registration strategies and confirm the presumed stability within the three test areas. The ability to detect stable areas is clearly essential when developing monitoring methods to identify areas exhibiting real change.

TABLE III. M3C2 differences (in terms of mean distances $(\mu)$ and standard deviations $(\sigma)$ ) between the single epoch and multi-epoch registration approaches comparing the SfM-based reconstructions in three stable areas (models compared: Cliff1 and Cliff2).

\begin{tabular}{ccccccccc}
\hline & \multicolumn{9}{c}{ Manual } & \multicolumn{4}{c}{ Manual + ICP } & \multicolumn{2}{c}{ Time-SIFT } & \multicolumn{2}{c}{ Time-SIFT+ICP } \\
\cline { 2 - 10 } $\begin{array}{c}\text { Stable } \\
\text { area ID }\end{array}$ & $\mu(m)$ & $\sigma(m)$ & $\mu(m)$ & $\sigma(m)$ & $\mu(m)$ & $\sigma(m)$ & $\mu(m)$ & $\sigma(m)$ \\
\hline 1 & -0.027 & 0.0462 & -0.001 & 0.009 & 0.008 & 0.011 & -0.001 & 0.008 \\
\hline 2 & 0.017 & 0.0392 & -0.0025 & 0.009 & 0.000 & 0.009 & 0.000 & 0.007 \\
\hline 3 & 0.029 & 0.0278 & 0.0021 & 0.008 & -0.003 & 0.017 & 0.000 & 0.013 \\
\hline
\end{tabular}

\section{Site 2: Quarry (Bardon Hill, UK)}

At the Quarry site a direct accuracy assessment of the georeferencing technique was practicable. Here, the positions of the two prisms (IDs: 103 and 104) measured with the total station were available and these were compared to the photogrammetric point cloud estimates to provide an initial check on accuracy. Specifically, the differences shown in Table IV correspond to the discrepancies between the prism positions computed from the photogrammetry (using the estimated position of the quadcopter) and their true position (computed through the survey). The 3D accuracy achieved for both prisms (103 and 104) is approximately $0.50 \mathrm{~m}$, corresponding to a relative error ratio of 1:1400 (for an average acquisition distance of $700 \mathrm{~m}$ ). The accuracies achieved along the single axes in the OSTN15 grid system for prisms 103 and 104 were approximately 0.51 and $0.48 \mathrm{~m}$ ( $x$ axis or easting), 0.12 and $0.24 \mathrm{~m}$ ( $y$ axis or northing) and -0.02 and $0.10 \mathrm{~m}$ ( $z$ axis or elevation), respectively.

Table IV. Discrepancies between the positions of the two prisms (103 and 104) computed from the total station survey and the photogrammetric project (Quarry7-UAV). Differences are indicated in terms of distance along individual axes (Easting, Northing and Elevation) and of total 3D distance.

\begin{tabular}{|c|c|c|}
\hline \multicolumn{3}{|c|}{ Differences: Total station survey - Photogrammetry } \\
\hline Target ID & 103 & 104 \\
\hline Easting $(m)$ & 0.510 & 0.476 \\
\hline Northing $(m)$ & 0.124 & 0.244 \\
\hline Elevation $(\mathrm{m})$ & -0.021 & 0.103 \\
\hline $3 D$ distance & 0.525 & 0.545 \\
\hline
\end{tabular}

The multi-epoch registration approach adopted for the quarry was able to relate all the ground-based imagery to the OSTN15 grid coordinate system, based on the known UAV imagery positions. A visual verification of the final orientation of the ground-based camera stations and 3D model alignment is illustrated in Fig. 8. However, it must be noted that Quarry4 and Quarry5 could not initially be co-registered, probably due to poor image contrast arising from poor weather and visibility conditions during photo acquisition (Table II). An example of this misalignment is presented in Fig. 9(a), that illustrates the absence of tie points found between photos of two epochs (Quarry4 and Quarry7-UAV). However, 
it was possible to overcome this issue by increasing the "SmartMatch point density" from the default 'medium' (Fig. 9(c)) to 'very high'. Although this parameter modification slowed the matching and processing time, the detection of many additional tie points (Fig. 9(b)) allowed alignment using the Time-SIFT approach for both the Quarry4 and Quarry5 datasets.

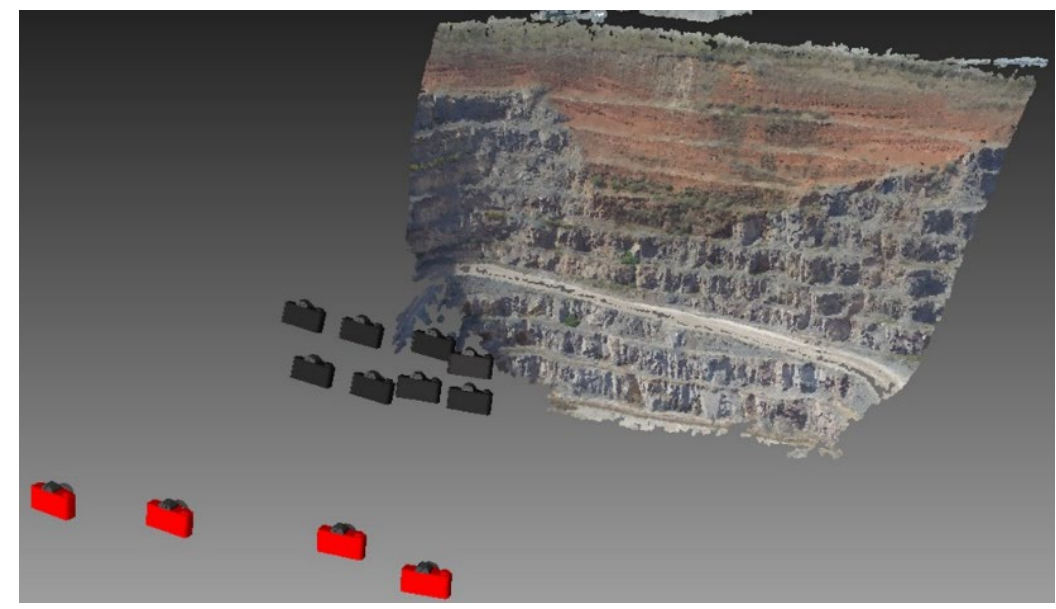

FIG. 8. Screenshot from PhotoModeler showing the position of the ground-based stations (four red cameras) orientated relative to the position of the UAV-based stations (eight cameras in black) through the Time-SIFT approach. The two aligning 3D models (obtained from the Quarry7 and UAV imagery) are also depicted.

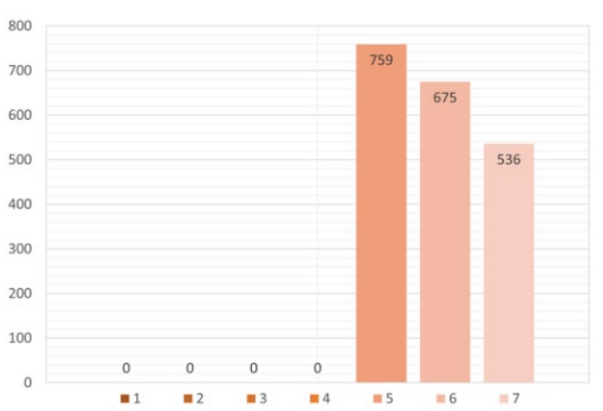

(a)

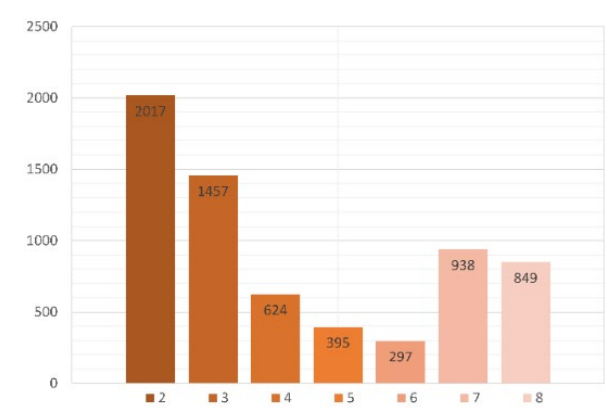

(b)

\begin{tabular}{lc}
\hline \multicolumn{2}{c}{ SmartMatch Project Options } \\
Feature Detection and Marking \\
\hline Point Density & $\begin{array}{c}\text { Low - } \\
\text { Extra High }\end{array}$ \\
\hline Good Overlap Count & 10 \\
\hline Match Quality Threshold & 0.5 \\
\hline Match Photos with Manual Reference & $\square$ \\
\hline Use Masks & $\square$ \\
\hline
\end{tabular}

(c)

FIG. 9. Example of connectivity by photo ( $x$ axis: photos ID; $y$ axis: number of tie points) after implementing the SmartMatch tool with different point densities. For this example two datasets were considered, namely Quarry4 (photos IDs: 1 - 4) and Quarry7-UAV (photos IDs: 5 - 8). (a) The first diagram shows the distribution of tie points between photo 8 (reference photo) and the other photos $(1-7)$, when a medium point density ('SmartMatch Project Option') is used. Photos $1-4$ (Quarry4) have no connectivity (zero tie points found) with the selected reference photo. (b) In the second diagram, photo 1 is used as the reference photo and a very high point density is used. In this case, all photos are connected ( $>\sim 300$ tie points found for each photo epoch through 8$)$ to the reference photo. (c) Example of settings controlling the SmartMatch tool in PhotoModeler. The point density parameter (in red) can be adjusted by the operator (from low to extra high).

The Gaussian distributions and spatial change maps in Fig. 10 were generated by estimating the M3C2-based distances between Quarry7 and Quarry7-UAV (the full extent of both point clouds was compared). These were aligned using the Time-SIFT approach with and without ICP. The statistics demonstrate that when using a Time-SIFT registration, the two clouds differ by $-0.008 \mathrm{~m}$ in terms of mean distance, with a standard deviation of $0.335 \mathrm{~m}$ (Fig. 10(a)). Applying the additional ICP algorithm produces only small changes to the distance statistics as shown in Fig. 10(b)). The high similarity of the two results 
implies that the Time-SIFT procedure has already achieved a satisfactory registration of the two clouds. Accordingly, the graphs (Gaussian distribution) and spatial distribution of discrepancies illustrated by the map of changes (Fig. 10) are comparable, reflecting similar performance of the compared registration strategies.

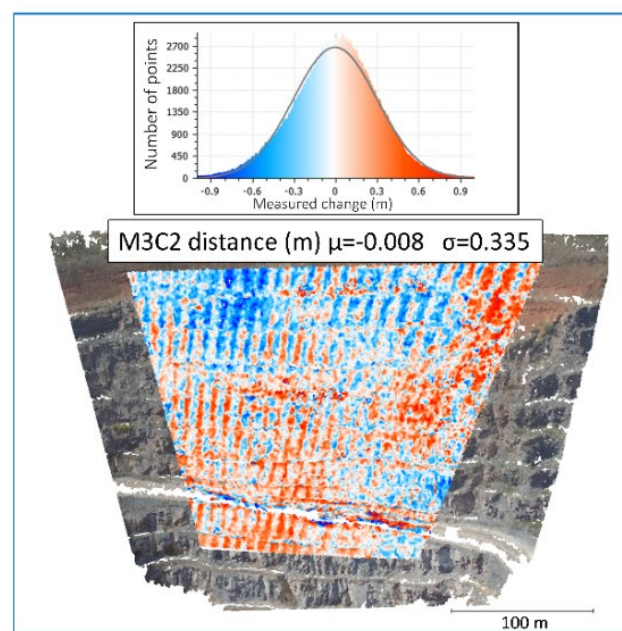

(a)

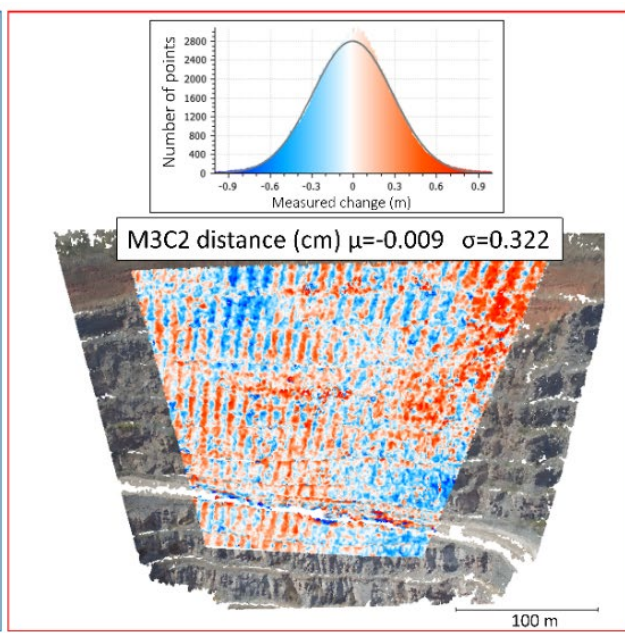

(b)

FIG. 10. Gaussian distribution and spatial distribution of $\mathrm{M} 3 \mathrm{C} 2$ error distances estimated between the UAV (Quarry7-UAV) and terrestrial (Quarry7) point clouds acquired on the same day when co-registered with

(a) Time-SIFT and (b) Time-SIFT+ICP strategies.

An additional source of information about the quality of the multi-epoch registration is provided in Table $\mathrm{V}$. The table shows the statistics in terms of mean distance and standard deviation of the $\mathrm{M} 3 \mathrm{C} 2$ distances estimated for a small section of the quarry $(15 \mathrm{~m} \mathrm{x} 15 \mathrm{~m})$ considered stable through the monitoring period (November 2018 - July 2019). For all comparisons carried out between the UAV cloud and the corresponding ground-based reconstructions (from Epoch1 to Epoch10), the results identify a clear improvement when the ICP algorithm is coupled to the Time-SIFT registration approach. In fact, a notable decrease in terms of mean distance is shown for all comparisons (Table V). The standard deviation values tend to improve slightly also. In fact, $\sigma$ values estimated using a TimeSIFT+ICP registration decrease when compared to those estimated with a Time-SIFT approach alone (differences range between 0.008 and $0.040 \mathrm{~m}$ ).

TABLE V. Quantitative assessment of the multi-epoch registration approach (Time-SIFT and Time-SIFT+ICP) by comparing a stable area between the UAV and ground-based reconstructions. The differences calculated with the M3C2 tool are indicated in terms of mean distances $(\mu)$ and standard deviations $(\sigma)$. See text for bold

\begin{tabular}{|c|c|c|c|c|}
\hline \multirow[b]{3}{*}{ Point clouds compared* } & \multicolumn{4}{|c|}{ Registration approach } \\
\hline & \multicolumn{2}{|c|}{ Time-SIFT } & \multicolumn{2}{|c|}{ Time-SIFT+ICP } \\
\hline & $\mu(m)$ & $\sigma(m)$ & $\mu(m)$ & $\sigma(m)$ \\
\hline Quarry7-UAV - Quarry1 & 0.158 & 0.247 & 0.029 & 0.239 \\
\hline Quarry7-UAV - Quarry2 & 0.480 & 0.207 & 0.079 & 0.188 \\
\hline Quarry7-UAV - Quarry3 & 0.392 & 0.212 & -0.089 & 0.191 \\
\hline Quarry7-UAV - Quarry4 & 0.118 & 0.232 & -0.020 & 0.213 \\
\hline Quarry7-UAV - Quarry5 & -0.377 & 0.229 & -0.066 & 0.215 \\
\hline Quarry7-UAV - Quarry6 & -0.271 & 0.191 & -0.028 & 0.183 \\
\hline Quarry7-UAV - Quarry7 & -0.242 & 0.217 & -0.070 & 0.208 \\
\hline Quarry7-UAV - Quarry8 & 0.470 & 0.193 & 0.0253 & 0.171 \\
\hline Quarry7-UAV - Quarry9 & 0.188 & 0.331 & 0.050 & 0.308 \\
\hline Quarry7-UAV - Quarry10 & -0.956 & 0.220 & 0.042 & 0.180 \\
\hline
\end{tabular}


Interestingly, the comparison between Quarry7 and Quarry7-UAV generated different statistics when the size of the compared point clouds differs. For example, comparing a full extent of the point clouds ( $250 \mathrm{~m}$ x $200 \mathrm{~m}$ ) (Fig. 10(b)) and a small portion of it (15 m x 15 $\mathrm{m})$ (Table V), results in terms of $\mu$ differ by approximately 0.23 and $0.06 \mathrm{~m}$, when a TimeSIFT and a Time-SIFT + ICP registration approach is used, respectively. It is suggested that the lower mean values derived in the full cloud comparison (Fig. 10), when compared to values in bold in Table $\mathrm{V}$, are probably due to the inevitable compensation of many more negative and positive deviations computed. In fact, the number of difference points generated for the full cloud and the small portion are approximately one million and six thousand, respectively.

\section{DISCUSSION}

The results of this work demonstrate that the assessed multi-epoch registration approach (Time-SIFT and Time-SIFT + ICP) has the potential to effectively co-register multitemporal SfM-MVS-based models; even when using multisource and multiperspective data, captured across widely varying spatial and temporal scales and under a range of weather and illumination conditions. This solution offers new possibilities into the automatic generation/alignment of 3D models using multitemporal and multisource/perspective images (Nex et al., 2015) without the need for GCPs. With the adoption of a Time-SIFT approach, commonly used registration strategies employed for 3D models registration purposes, such as identifying appropriate and sufficient stable features and/or defining the coordinates of well-distributed photogrammetric targets in the study area (Westoby et al., 2012; Esposito et al., 2017) are not required. This is particularly advantageous when time, equipment costs and expertise required to establish GCPs are considered.

The Cliff work revealed that aligning point clouds by manually identifying the same stable features and adopting a manual (point-pair tool) registration is time consuming, operator-dependent and can be inaccurate. This is due to a range of challenges, including: (i) ability of the operator to select the same paired well-distributed features for each epoch; (ii) dissimilarity in point reconstruction and distribution on paired clouds and (iii) stability of chosen features. Coupling the manual procedure to the ICP algorithm has the advantage of producing better alignments (Parente et al., 2019). However, when working with the ICP algorithm on multitemporal point clouds, an operator is still required to manually clean part of the 3D reconstruction (for example removing change areas - for example Turner et al., 2015) and avoid significant errors due to poor registration (Lague et al., 2013). Furthermore, an initial manual coarse registration must be carried out before applying the ICP (for example Micheletti et al., 2014) in order to obtain reliable transformation parameters. Thus, the required operator-based operations limit automation and time efficiency of a SfM-based change detection system.

A SfM-MVS-based multitemporal monitoring routine combined with a Time-SIFT registration strategy can achieve improved results. This registration procedure enabled the three previously described challenges encountered during manual registration to be overcome. In fact, the Time-SIFT registration is significantly more reliable as thousands of well-distributed tie points are identified and potential matching points that have changed (for example due to natural processes) are removed automatically. This is clearly advantageous when compared to the time-consuming and basic manual procedures previously described, where it is impracticable to identify more than just several GCPs. This finding resonates with the work of Micheletti et al. (2014) who suggest the importance of identifying a high number of GCPs to reduce alignment error.

The Cliff study case further demonstrates that 3D models automatically co-registered with the Time-SIFT approach can directly produce alignments that are comparable to the manual+ICP solution, revealing the potential for similar applications. Analyses with single epoch and multitemporal Cliff datasets showed a strong agreement between the classic (manual $+\mathrm{ICP}$ ) and Time-SIFT $+\mathrm{ICP}$ registration approaches. Specifically, the average statistics (estimated for the Cliff study site) highlighted an average measured error that differs by only $0.002 \mathrm{~m}$ when comparing the manual+ICP to the Time-SIFT+ICP registration outputs. In Al-Rawabdeh et al. (2016), multitemporal photogrammetric point clouds generated from sets of images captured solely from a UAV with a camera-object 
distance is $25-30 \mathrm{~m}$ (GSD comparable to the UAV-based acquisition of the Cliff study site), were registered adopting a similar procedure to the Time-SIFT approach used in this paper. In their study, the mean distance and standard deviations calculated and compared within stable area surfaces were $0.025 \mathrm{~m}$ and $0.027 \mathrm{~m}$, respectively. The multi-epoch approach used in this work demonstrated the possibility to achieve a better alignment accuracy, as evidenced by lower statistical values (Table III).

Analysis of the M3C2 maps in Fig. 7 demonstrated that the sharp changes in Fig. 7(a), where there is a sudden transition between red and blue points, is due to a change of about $0.02 \mathrm{~m}$ (from about $0.01 \mathrm{~m}$ (in red) to about $-0.01 \mathrm{~m}$ (in blue)) between neighbouring points. For the other representations (Fig. $7(\mathrm{~b}, \mathrm{c}$ and d)) such change is more gradual (neighbouring points differ by approximately $0.001 \mathrm{~m}$ ). It is suggested that seemingly small decisions made during the manual procedure inevitably influence the results quite considerably. The number and distribution of the selected tie-points, and the ability to accurately measure them, must cause small rotations and hence a slight bias in the consequent datums used for comparison.

The statistics describing the quality of the multi-epoch registration strategies for the Quarry work highlighted that the precision achieved when comparing the terrestrial and UAV-based cloud is in the range $0.320-0.330 \mathrm{~m}$ (mean distance: $-0.008 \mathrm{~m}$ ). It was noted that comparing multitemporal terrestrial reconstructions and the UAV model (considering only a small stable zone), the average precision improves to a value of between 0.210 and $0.230 \mathrm{~m}$ (mean distance: $-0.009 \mathrm{~m}$ ). Although not directly comparable, similar results were broadly achieved by Vargo et al (2017). In the latter, in order to validate the SIFT-based registration they compared a surface elevation survey collected using a Trimble R8 receiver (25th -27th March 2016) with a SfM-based DEM (30th March 2016). Such comparison produced a standard deviation of about $0.50 \mathrm{~m}$ and mean distance of about $-0.01 \mathrm{~m}$. Regarding the spatial distribution of $\mathrm{M} 3 \mathrm{C} 2$ error distances displayed in Fig. 10, the presence of a noticeable spatial pattern (characterised by parallel stripes) revealed the presence of systematic errors which may have been introduced by the acquisition of poorly convergent UAV-based images (James and Robson, 2014).

Results (in terms of mean distance and standard deviation) of the Quarry study case are commensurate with those achieved for the Cliff site. Given the relative image resolution and project scale, the Quarry study produced better results, even though fewer UAV images were available when compared to the Cliff site. The combination of UAV-based acquisition, with an imaging distance of almost half that of the terrestrial imagery, has helped in this case. In both study cases, the standard deviation values are moderately lower when a Time-SIFT + ICP registration is used, demonstrating that inclusion of the ICP algorithm can further refine the co-registration at a range of scales. While the works of Filhol et al. (2019) and Cook and Dietze (2019) did not apply this refinement to their SIFTbased registrations, Kromer et al. (2019) implemented an automated alignment procedure for ground-based photogrammetric models which included the ICP step. However, in the latter work, an assessment of their registration procedure is not reported and so full comparison with the Time-SIFT+ICP approach described here is not possible.

For the Quarry site the authors considered adopting a fixed-camera set-up that has the critical advantage of generating photogrammetric datasets in the same local reference system (Travelletti et al., 2010). Although this configuration is advantageous in a controlled environment (for example Parente et al., 2019), the current work highlights that a fixed camera approach is often impracticable in the field. In agreement with Armstrong et al. (2018), it is recognised that a range of unpredictable factors, including extreme weather conditions, wildlife and soil deformations, can destabilise one or more camera stations over the monitoring period. Furthermore, the Quarry work demonstrated that, in addition to natural causes, vandalism can be regarded as another factor that may affect ground-based photogrammetric projects, even when the studied site has supposedly restricted access.

For photogrammetric projects with both variable camera stations and without GCPs, this paper has presented an innovative co-registration solution. The use of the tracking mode function of a robotic total station demonstrates the ability to generate a UAV-based photogrammetric model georeferenced to the OSTN15 grid coordinate system with an accuracy of about $0.50 \mathrm{~m}$. The use of such a reference photogrammetric model in combination with the multi-epoch Time-SIFT workflow enabled the location and orientation of the ground-based set of images to be determined. With this approach, all epoch image sets collected for the Quarry (over a time span of nine months) were aligned 
to a common spatial reference system. Thus, with the proposed approach, the timeconsuming tasks to install/identify GCPs and strategies for ensuring stationary camera stations in the field (such as Armstrong et al., 2018) or adopting camera motion or orientation corrections (for example Zitova and Flusser, 2003) are not required. It is recognised that the adoption of the proposed direct georeferencing approach is not a suitable solution for low-budget applications. Also, the temporal accuracy of audio communication (below one second) for camera and positioning synchronisation is comparatively poor compared to conventional RTK-dGNSS systems (Turner et al., 2014). However, the recent availability on the market of UAVs equipped with survey-grade RTK systems (for example Ekaso et al., 2020) can facilitate the implementation of a quicker and even more convenient (in terms of price, time and logistics) direct georeferencing process.

This work has showed that aligning point clouds using the Time-SIFT registration strategy is effective for images captured under a range of conditions (variable weather/illumination conditions, multisource/perspective, multiscale and multitemporal). The authors expected to obtain a reduction in the quality of the alignment when using point clouds derived from epochs captured during poor weather and atmospheric conditions (such as the cloudy and foggy days in the Quarry study case), resulting from a reduced number of detected tie-points. However, through minor modification of the parameters used to compute the multitemporal tie points, results showed that all alignments achieved comparable precision (in terms of standard deviation, as shown in Table V). Furthermore, a clear relationship between weather/illumination conditions (Table II) and statistics (Table V) is not discernible. In Vargo et al. (2017), the implementation of the Time-SIFT strategy with datasets collected in adverse weather conditions and reduced study area depicted in the photographs led to a misorientation of the models (thus some photos were not aligned correctly). Similarly, some Quarry image sets were initially misaligned due to the absence of common tie points with the reference model (Fig. 9). Possibly, many well distributed and stable features identified across the study area remain essential for optimising alignments when using variable camera stations with the Time-SIFT approach. This finding agrees with other Time-SIFT-based works, which suggested either stable landmarks or stationary cameras to ensure the correct co-registration of point clouds (Filhol et al., 2019). Consequently, one should manage to image an area of interest where stable zones are dominant to ensure a sufficient number of multitemporal tie points (Feurer and Vinatier, 2018, Cook and Dietze, 2019). Still, it is believed that further work must be conducted to better understand the effects of distribution of tie points and to quantify the minimum number of such points necessary.

\section{CONCLUSION}

Generic SfM-MVS capabilities have been exploited and assessed to implement an automated Time-SIFT approach. The study was conducted on two different sites of widely differing scales and using a range of data collection and registration strategies (such as GCPs/no GCPs over the study area, and combinations of imagery captured from different platforms and sensors). The results reveal that a multi-epoch registration approach (including both Time-SIFT and Time-SIFT + ICP) can achieve an accurate alignment between multitemporal point clouds when compared to traditionally used registration methods (manual and manual $+\mathrm{ICP}$ ).

The primary contribution of this registration approach is the automated capability to minimise geometric misalignment among multitemporal and multiscale photogrammetric reconstructions generated with both terrestrial and UAV datasets (images acquired with a range of different camera tilts), even under different weather/illumination conditions. The Time-SIFT strategy alone can already provide an acceptable alignment; however, the inclusion of an ICP algorithm produced a further moderate improvement in co-registration.

Additionally, the Quarry work provided the opportunity to investigate a georeferencing approach that fused UAV-based image capture with a robotic total station. This approach allowed the georeferencing of 3D models to an absolute coordinate system, achieving a relative error of 1:1400. This solution can be adopted for challenging environments where the deployment of GCPs is impracticable. However, it is recognised that combining the Time-SIFT method with the new class of UAVs equipped with RTK 
GNSS systems creates a more robust, cost-effective and time-saving solution for a range of future photogrammetric applications.

Specifically, key findings of the investigated Time-SIFT approach highlighted in the current work include:

(1) High-quality alignment is achievable for a range of scales and landscape types, even when using aerial and terrestrial images acquired with different type of cameras (DSLR and in-built UAV camera).

(2) All point clouds are generated directly in a common reference system, even with images acquired under a range of weather/illumination conditions (different times and seasons).

(3) The adoption of an ICP algorithm to point clouds already referenced using Time-SIFT improves the quality of the alignment.

(4) If a reference photogrammetric dataset is available, there is no need for the user to upload further external information (no need for GCPs on the study site) or to ensure/assume that camera stations are truly static.

(5) The investigated alignment strategy is time-saving and reliable, as potentially thousands of points are used in the registration procedure with unstable and dynamic features (changing points) automatically removed.

Tests were conducted solely in PhotoModeler, but the approach can easily be implemented in other SfM software packages. Furthermore, the procedure can be fully automated using commonly used programming languages (for example, using Python, and MATLAB). Thus, it is believed that the Time-SIFT and Time-SIFT+ICP strategies represent suitable registration solutions to implement fully automated monitoring system based on the use of SfM-MVS photogrammetry.

We suggest that other further work should be addressed to fully investigate the capabilities of the Time-SIFT approach and, in particular, to better understand the relationship between the quality of the alignment and number and distribution of tie points. This may be an important area for future research about Time-SIFT methods and its derivatives, such as Time-SIFT+ICP proposed in this paper.

\section{ACKNOWLEDGEMENTS}

The authors are grateful to the Aggregate Industries UK Limited for their consent to access the Bardon Hill quarry site, in particular to Jack Manuel (Geotechnical Engineering) and Kris Patrick (Quarry Supervisor) for their support and assistance in the field. The authors also thank Nick Rodgers, surveying technician at Loughborough University, for logistical support and data collection.

\section{REFERENCES}

Al-Rawabdeh, A., Al-Gurrani, H., Al-Durgham, K., Detchev, I., He, F., El-Sheimy, N. and Habib, A., 2016. A robust registration algorithm for point clouds from UAV images for change detection. International Archives of Photogrammetry, Remote Sensing and Spatial Information Sciences, 41(B1): 765-772.

Armstrong, L., LACElle, D., Fraser, R. H., KoKelJ, S. and KNUDBY, A., 2018. Thaw slump activity measured using stationary cameras in time-lapse and structure-from-motion photogrammetry. Arctic Science, 4(4): 827845 .

BESL, P. J. and MCKAY, N. D., 1992. Method for registration of 3-D shapes. SPIE, 1611: 586-606.

Brunier, G., Fleury, J., ANTHONY, E. J., GARDEL, A. and DussouilleZ, P., 2016. Close-range airborne structurefrom-motion photogrammetry for high-resolution beach morphometric surveys: examples from an embayed rotating beach. Geomorphology. 261: 76-88.

Burton, W., 1622. The Description of Leicester Shire. Containing Matters of Antiquitye, Historye, Armorye, and Geneology. John White, London, England. 320 pages.

CARNeY, J. N., 2010. Guide to the geology of Mount St. Bernard, Charnwood Lodge, Warren Hills and Bardon Hill, Charnwood Forest. British Geological Survey. Open Report OR/10/044. 26 pages. http://nora.nerc.ac.uk/id/eprint/12098/ [Accessed: 16th January 2020].

Chandler, J. H. and BuCKLEY, S., 2016. Structure from motion (SfM) photogrammetry vs terrestrial laser scanning. Section 20.1 in Carpenter, M. B. and Keane, C. M. (Eds.): Geoscience Handbook: AGI Data Sheets, 5th edition. American Geosciences Institute, Alexandria, Virginia, USA.

CHEN, H. R. and TSENG, Y. H., 2016. Study of automatic image rectification and registration of scanned historical aerial photographs. International Archives of Photogrammetry, Remote Sensing and Spatial Information Sciences, 41(B8): 1229-1236.

CloudCompare, 2019. CloudCompare Version 2.10.2 (Zephyrus) GPL Software. www.cloudcompare.org [Accessed: $10^{\text {th }}$ December 2019]. 
CoOK, K. L. and Dietze, M., 2019. Short communication: a simple workflow for robust low-cost UAV-derived change detection without ground control points. Earth Surface Dynamics, 7: 1009-1017.

Cucchiaro, S., Maset, E., Cavalli, M., Crema, S., Marchi, L., Beinat, A. and Cazorzi, F., 2020. How does co-registration affect geomorphic change estimates in multi-temporal surveys? GIScience \& Remote Sensing, 57(5): 22 pages.

Eltner, A., Kaiser, A., Castillo, C., Rock, G., Neugirg, F. and Abellán, A., 2016. Image-based surface reconstruction in geomorphometry-merits, limits and developments. Earth Surface Dynamics, 4(2): 359-389.

EKASO, D., NEX, F. and KeRLE, N., 2020. Accuracy assessment of real-time kinematics (RTK) measurements on unmanned aerial vehicles (UAV) for direct geo-referencing. Geo-Spatial Information Science, 23(2): 17 pages.

Esposito, G., SAlvini, R., MAtano, F., SACChi, M., DAnZi, M., Somma, R. and Troise, C., 2017. Multitemporal monitoring of a coastal landslide through SfM-derived point cloud comparison. Photogrammetric Record, 32(160): 459-479.

FEURER, D. and VINATIER, F., 2018. Joining multi-epoch archival aerial images in a single SfM block allows 3-D change detection with almost exclusively image information. ISPRS Journal of Photogrammetry and Remote Sensing, 146: 495-506.

Feurer, D., Bemis, S., Coulouma, G., Mabrouk, H., Massuel, S., Barbosa, R. V., Thomas, Y., Ammann, J. and VINATIER, F., 2020. Time-SIFT: a frugal method for leveraging multi-temporal photogrammetric data without ancillary data, EGU General Assembly 2020, Online, 4-8 May 2020, EGU2020-10187. https://meetingorganizer.copernicus.org/EGU2020/EGU2020-10187.html

Filhol, S., Perret, A., Girod, L., Sutter, G., Schuler, T. V. and Burkhart, J. F., 2019. Time-lapse photogrammetry of distributed snow depth during snowmelt. Water Resources Research, 55(9): 7916-7926.

FOX, A. J. and CZIFERSZKY, A. Unlocking the time capsule of historic aerial photography to measure changes in Antarctic peninsula glaciers. Photogrammetric Record, 23(121): 51-68.

Gruen, A. and BeYer, H. A., 2001. System calibration through self-calibration. Chapter 7 in Calibration and Orientation of Cameras in Computer Vision (Eds. A. Gruen and T. S. Huang). Springer Series in Information Sciences, Vol. 34. Heidelberg, Germany. 229 pages: 163-193.

JAMES, M. R., and ROBSON, S., 2012. Straightforward reconstruction of 3D surfaces and topography with a camera: Accuracy and geoscience application. Journal of Geophysical Research, 117: 1-17.

JAMES, M. R., and ROBSON, S., 2014. Mitigating systematic error in topographic models derived from UAV and ground-based image networks. Earth Surface Processes and Landforms, 39 (10): 1413-1420.

James, M. R., Chandler, J., Eltner, A., Fraser, C., Miller, P., Mills, J., Noble, T., Robson, S. and Lane, S., 2019. Guidelines on the use of structure from motion photogrammetry in geomorphic research. Earth Surface Processes and Landforms, 44(10): 2081-2084.

JAO, F. J., ChU, H, J. and TsenG, Y. H., 2014. Historical images registration and land-use land-cover change analysis. Environments, 1: 181-189.

Kromer, R., Walton, G., Gray, B. and Lato, M., 2019. Development and optimization of an automated fixedlocation time lapse photogrammetric rock slope monitoring system. Remote Sensing, 11(16), 1890.

Lague, D., Brodu, N. and LerouX, J., 2013. Accurate 3D comparison of complex topography with terrestrial laser scanner: application to the Rangitikei canyon (N-Z). ISPRS Journal of Photogrammetry and Remote Sensing, 82: 10-26.

Lowe, D. G., 2004. Distinctive image features from scale-invariant keypoints. International Journal of Computer Vision, 60(2): 91-110.

Micheletti, N., Chandler, J. H. and LANE, S. N., 2014. Investigating the geomorphological potential of freely available and accessible structure-from-motion photogrammetry using a smartphone. Earth Surface Processes and Landforms, 40(4): 473-486.

Mosbrucker, A. R., Major, J. J., Spicer, K. R. and Pitlick, J., 2017. Camera system considerations for geomorphic applications of SfM photogrammetry. Earth Surface Processes and Landforms, 42(6): 969-986.

NeX, F., GERKE, M., REMONDINO, F., PrZYBILla H.-J., BÄUMKER, M. and ZuRHORST, A., 2015. ISPRS Benchmark for multi-platform photogrammetry. ISPRS Annals of Photogrammetry, Remote Sensing and Spatial Information Sciences, 2(3/W4): 135-142.

PARENTE, L., CHANDLER, J. and DiXON, N., 2019. Optimising the quality of an SfM-MVS slope monitoring system using fixed cameras. Photogrammetric Record, 34(168): 404-427.

PhotoModeler, 2019. PhotoModeler Software Version 2019.1.2.2469 (Premium). User guide available at: https:/www.photomodeler.com/downloads/OnlineHelp/index.html\#!welcometophotomodeler.htm [Accessed 10th December 2019].

Prosdocimi, M., Calligaro, S., Sofia, G., Dalla Fontana, G. and Tarolli, P., 2015. Bank erosion in agricultural drainage networks: new challenges from structure-from-motion photogrammetry for post-event analysis. Earth Surface Processes and Landforms, 40(14):1891-1906.

Scaioni, M., Feng, T., Barazzetti, L., Previtali, M. and Roncella, R., 2015. Image-based deformation measurement. Applied Geomatics, 7: 75-90.

Seitz, S.M., Curless, B., Diebel, J., Scharstein, D. and Szeliski, R., 2006. A comparison and evaluation of multi-view stereo reconstruction algorithms. IEEE Computer Society Conference on Computer Vision and Pattern Recognition, New York, USA. 1: 519-528.

SMith, M. W., CARrivick, J. L. and QuinCEY, D. J., 2015. Structure from motion photogrammetry in physical geography. Progress in Physical Geography, 40(2), 247-275.

Snavely, N. N., Seitz, S. M. and Szeliski, R., 2006. Photo Tourism: Exploring Photo Collections in 3D. ACM Transactions on Graphics, 25(3): 835-846.

Thoeni, K., Giacomini, A., Murtagh, R. and Kniest, E., 2014. A comparison of multi-view 3D reconstruction of a rock wall using several cameras and a laser scanner. International Archives of Photogrammetry, Remote Sensing and Spatial Information Sciences, 40(5): 573-580.

Travelletti, J., Malet, J., SchmittBuhl, J., Toussaint, R., Bastard, M., Delacourt, C., Allemand, P. and VAN DAM, D. B., 2010. Multi-temporal terrestrial photogrammetry for landslide monitoring. Mountain Risks International Conference, Florence, Italy. Pages 183-191.

TURNER, D., LUCIEER, A., and DE JONG, S.M., 2015. Time series analysis of landslide dynamics using an unmanned aerial vehicle (UAV). Remote Sensing 7(2): 1736-1757. 
TURNER, D., LuCIEER, A. and WALLACE, L., 2014. Direct georeferencing of ultrahigh-resolution UAV imagery. IEEE Transactions on Geoscience and Remote Sensing, 52(5): 2738-2745.

VARGo, L. J., ANDERSON, B. M., Horgan, H. J., Mackintosh, A. N., LORRey, A. M. and Thornton, M., 2017. Using structure from motion photogrammetry to measure past glacier changes from historic aerial photographs. Journal of Glaciology, 63(242): 1105-1118.

Westoby, M. J., Brasington, J., Glasser, N. F., HAMBrey, M. J. and ReynOldS, J. M., 2012. "Structure-fromMotion" photogrammetry: A low-cost, effective tool for geoscience applications. Geomorphology, 179: 300314

ZANG, Y., YANG, B., LI, J. and GUAN, H. 2019. An accurate TLS and UAV image point clouds registration method for deformation detection of chaotic hillside areas. Remote Sensing, 11(6), 647, 1-26.

ZitOVA, B., and FluSSER, J., 2003. Image registration methods: a survey. Image and Vision Computing, 21(11), 977-1000. 